\title{
Chinese herb extract improves liver steatosis by promoting the expression of high molecular weight adiponectin in NAFLD rats
}

\author{
XUDONG LIU, WANGXIA TONG, XIAOFANG ZHAO, HONGXING ZHANG, YANFANG TANG and XIN DENG
}

\author{
Department of Liver Disease, Ruikang Hospital Affiliated to Guangxi University of \\ Traditional Chinese Medicine, Nanning, Guangxi 530011, P.R. China
}

Received July 2, 2016; Accepted April 27, 2017

DOI: $10.3892 / \mathrm{mmr} .2017 .7284$

\begin{abstract}
High molecular weight (HMW) adiponectin (APN) is closely correlated with the development of fatty liver and is modulated by the Akt/forkhead box protein O1 (FOXO1) pathway through disulfide-bond A oxidoreductase-like protein (DsbA-L). The Chinese herb extract, QSHX, is used to treat liver diseases. The present study investigated the effects of QSHX on non-alcoholic fatty liver disease (NAFLD) and its underlying mechanism. A rat model of NAFLD was established by feeding of a high-fat and high-sugar diet for 20 weeks. From week 13, the rats were administered with QSHX, or saline as a control, for 8 weeks. The liver function, blood fat and plasma APN were measured using a radioimmunoassay. The hepatic tissue score was measured following staining for pathology. The expression and activities of Akt, FOXO1, DsbA-L and HMW APN in the adipose tissue and primary adipocytes of the rats were measured using western blot analysis. It was found that QSHX significantly decreased the body weight, liver index, and serum levels of aspartate aminotransferase, alanine aminotransferase and triglyceride; and increased the serum level of APN in the NAFLD rats. Following 8 weeks of treatment with QSHX, the hepatic steatosis in the liver tissue improved and the score of hepatic steatosis was significantly decreased. The results of the western blot analysis indicated that QSHX promoted the expression of DsbA-L and HMW APN, and reduced the expression levels of phosphorylated FOXO1 and FOXO1 in adipose tissue and primary adipocytes. It was concluded that QSHX reduced hepatic steatosis by promoting the expression of HMW APN and DsbA-L, which may have been induced by inhibiting the activation and expression of FOXO1 in adipocytes.
\end{abstract}

Correspondence to: Dr Xudong Liu, Department of Liver Disease, Ruikang Hospital Affiliated to Guangxi University of Traditional Chinese Medicine, 10 Huadong Road, Nanning, Guangxi 530011, P.R. China

E-mail: 1xdlhx@163.com

Key words: Chinese herb extract, Akt/forkhead box protein O1, disulfide-bond A oxidoreductase-like protein, adiponectin, non-alcoholic fatty liver disease

\section{Introduction}

Non-alcoholic fatty liver disease (NAFLD) is hepatic steatosis caused by the accumulation of excess triglyceride (TG) in the liver. Patients with NAFLD usually exhibit hepatocyte injury and inflammation, which is termed non-alcoholic steatohepatitis (NASH) (1). NASH is a global chronic liver disease, which is becoming more common, and is closely associated with diabetes and obesity. According to epidemiological findings (2), at least 1.46 billion adults in the world are obese; in the USA, $\sim 6,000,000$ of those with obesity develop NASH and 600,000 suffer NASH-associated hepatic cirrhosis. Increasing data indicate that NAFLD/NASH has high morbidity rates in the Middle East, Far East, Africa, the Caribbean, USA and Latin America (2). These problems are a significant challenge to human health, however; there is no ideal therapeutic strategy for NAFLD/NASH.

It is reported that adiponectin (APN) is the only cytokine of benefit in NAFLD and its major active form is high molecular weight (HMW) APN. The polymerisation of HMW APN is modulated predominantly by disulfide-bond A oxidoreductase-like protein (DsbA-L) (3). A previous study indicated that the expression of DsbA-L in adipose cells was regulated by the Akt/forkhead box protein O1 (FOXO1) pathway (4). The extracts of Chinese herbs, termed QSHX, have been used in several diseases and have shown satisfactory effects, particularly for liver diseases in China (5). The present study investigated the effects of QSHX on liver steatosis in NAFLD rats and its underlying mechanism.

\section{Materials and methods}

Chinese herb extract preparation. Dried, sliced crude herbs (6 g Bupleurum falcatum, $6 \mathrm{~g}$ Salvia miltiorrhiza, $6 \mathrm{~g}$ rhubarb, $6 \mathrm{~g}$ lotus leaf, $9 \mathrm{~g}$ capillary Artemisia, $15 \mathrm{~g}$ rhizome polygoni cuspidate and $15 \mathrm{~g}$ gynostemma pentaphyllum) were decocted twice using tap water (40 min each). The decocted solution was filtered through a $150 \mu \mathrm{m}$ gauze and the filtrate was then concentrated to a mass using a vacuum desiccator at $70^{\circ} \mathrm{C}$. The sediment was dried into powder and the powder was dissolved in $25 \mathrm{ml}$ water with $0.9 \%$ saline.

NAFLD model establishment and animal treatment. All experiments were approved by the Animal Care and Use 
Committee of Ruikang Hospital Affiliated to Guangxi University of Traditional Chinese Medicine (Nanning, China). A total of 40 adult male Sprague-Dawley (SD) rats of SPF grade (180-200 g) were provided by the Experimental Animal Center of Guangxi Medical University [Certificate of quality no. SCXK (Gui) 2013-0002] and were housed in a restricted access room with controlled temperature $\left(23^{\circ} \mathrm{C}\right)$ and a $12-\mathrm{h}$ light/dark cycle. The animals were housed for 3 days prior to experiments, and were randomly divided into a normal control group $(n=15)$, model control group $(n=15)$ and treatment group $(n=10)$. The rats in the normal control group were fed a normal diet, whereas the rats in model control group and treatment group were fed with a high-fat and high-sugar diet $(72.8 \%$ normal diet $+10 \%$ lard $+10 \%$ sucrose $+2 \%$ cholesterol $+5 \%$ yolk powder $+0.2 \%$ propylthiouracide) for 20 weeks. The successful establishment of the animal model was confirmed at week 12 by examination of liver pathology in the rats from the normal control group and model control group $(n=5$ for each group). Water was freely available to the animals during the experiment.

From week 13, the rats in the treatment group were administered intragastrically with dissolved QSHX. The dose administered was $1 \mathrm{ml}$ each day per rat. The rats in the normal control group and model group were administrated intragastrically with $1 \mathrm{ml}$ water with $0.9 \%$ saline. The administration was performed daily for 8 weeks. The body weights of the rats was measured prior to and following treatment. At the end of experiment, the wet weight of the liver was measured to calculate the liver index using the following equation: Liver index $=$ liver wet weight/body weight $\mathrm{x} 100 \%$.

Serum sample preparation and examination. Following 8 weeks of treatment, $5 \mathrm{ml}$ blood was collected from the abdominal aorta into a vacuumed anticoagulation tube under anesthesia with $10 \%$ chloral hydrate $\left(3.5 \mathrm{ml} \cdot \mathrm{kg}^{-1}\right)$, which was placed standing for $10 \mathrm{~min}$ and centrifuged for $20 \mathrm{~min}$ at $1,000 \mathrm{x} g$ at room temperature to collect the serum. The serum was stored in $-20^{\circ} \mathrm{C}$ until the experiments. A TG kit (Dongou Diagnostic Production, Ltd., Zhejiang, China), alanine aminotransferase (ALT)/glutamic-pyruvic transaminase kit (Nanjing Jiangcheng Bioengineering Institute, Nanjing, China) and aspartate aminotransferase (AST)/glutamic oxaloacetic transaminase kit (Nanjing Jiancheng Bioengineering Institute) were used to examine the respective parameters according to the manufacturer's protocol. The serum level of APN was measured using an enzyme-linked immunosorbent assay (ELISA) kit (ab108784, Cambridge, UK) according to the manufacturer's protocol.

Pathological examination. The animals were sacrificed by cervical dislocation, following which two sections of hepatic tissue were collected from the same site of the right lobe, fixed in $4 \%$ formaldehyde, embedded in paraffin, sectioned and stained with hematoxylin and eosin (HE). Under an optical microscope, 3 fields (magnification, x200) were randomly selected from each slide to determine the level of hepatic steatosis according to the following scale: 0 , no hepatic steatosis; $1,<25 \%$ degenerated adipocytes; $2,26-50 \%$ degenerated adipocytes; $3,51-75 \%$ degenerated adipocytes; $4,>75 \%$ degenerated adipocytes.
Protein expression in fat tissues of rats. The protein from the epididymis fat pad was extracted according to the protocol of MinuteTM Adipose Tissue Fractionation kit (AF-023; Invent Biotechnologies, Inc., Plymouth, MN, USA). The concentration of protein was measured using a bicinchoninic acid protein assay kit (Beyotime Insitute of Biotechnology, Shanghai, China). Subsequently, samples of $100 \mathrm{~g}$ protein were diluted 1:4 with $5 \mathrm{X}$ SDS, denatured at $99^{\circ} \mathrm{C}$ for $5 \mathrm{~min}$ and cooled in an ice bath. The samples were then subjected to $8 \%$ SDS-PAGE and transferred onto a PVDF membrane, which was incubated with primary antibodies diluted with $5 \%$ skim milk overnight at $4{ }^{\circ} \mathrm{C}$. The primary antibodies were as follows: GADPH (cat. no. AP0063; 1:5,000; Bioworld Technologies, Inc., St. Louis Park, MN, USA), Akt (cat. no. ab9150; 1:2,000; Abcam, Cambridge, UK), phosphorylated (p)-Akt (cat. no. 2965; 1:1,000; Cell Signaling Technology, Inc., Danvers, MA USA), FOXO1 (cat. no. 2880; 1:10,00; Cell Signaling Technology, Inc.), p-FOXO1 (cat. no. 9461; 1:1,000; Cell Signaling Technology, Inc.), DsbA-L (cat. no. ab92819; 1:2,000; Abcam, Cambridge, UK) and APN (cat. no. bs-0471R; 1:300; Bioss, Beijing, China). The PVDF membrane was then rinsed twice with TBST (5 min each), and incubated with secondary antibodies labelled with HRP (cat. no. BS13278; 1:5,000; Bioworld Technologies, Inc.) for $2 \mathrm{~h}$ at room temperature. The bands were developed using the chemiluminescence method and scanned onto film for image analysis.

Primary cell isolation, culture and differentiation. In order to determine the effect of QSHX on HMW APN, primary adipocytes of rats were used. Male SD rats in the normal control group were fed for 20 weeks and were sacrificed by cervical dislocation. The intra-abdominal fat pads were excised and the preadipocytes were isolated. Collagenase digestion was performed at $37^{\circ} \mathrm{C}$ for $1 \mathrm{~h}$ and the harvested cells were cultured in DMEM (Thermo Fisher Scientific, Inc., Waltham, MA, USA) supplemented with $2 \mathrm{mM}$ penicillin-streptomycin $(100 \mathrm{U} / \mathrm{ml})$ and $10 \%$ fetal bovine serum (FBS, Hyclone; GE Healthcare Life Sciences, Logan, UT, USA) at $37^{\circ} \mathrm{C}$ and $5.0 \%$ $\mathrm{CO}_{2}$. The medium was routinely replaced every 2-3 days until the cells reached a confluent state. The preadipocytes were cultured in 12-well plates at $2 \times 10^{5}$ cells/well. The induction of differentiation was performed on day 0 using DMEM supplemented with penicillin-streptomycin (100 U/ml), 10\% FBS, $0.5 \mathrm{mM}$ IBMX, $1.0 \mu \mathrm{M}$ dexamethasone and $5 \mu \mathrm{g} / \mathrm{ml}$ insulin at $37^{\circ} \mathrm{C}$ in $5.0 \% \mathrm{CO}_{2}$. On the subsequent 2 days, the cells were cultured in similar medium but without IBMX or dexamethasone. After 2 days, the cells medium was replaced with DMEM containing $10 \% \mathrm{FBS}$ and $100 \mathrm{U} / \mathrm{ml}$ penicillin-streptomycin. Experiments were performed on days 8-10. Lipid accumulation was examined using Oil-Red-O staining by conventional light microscopy, with stained cells considered to be mature adipocytes for use in the subsequent assays.

Primary adipocyte assays. The mature primary adipocytes were obtained using the method described above and cultured in DMEM containing $10 \% \mathrm{FBS}$ and $100 \mathrm{U} / \mathrm{ml}$ penicillin-streptomycin. The QSHX powder was dissolved in DMEM and was passed through a $0.22 \mu \mathrm{m}$ filter. Following culture of the primary adipocytes for $24 \mathrm{~h}$ with DMEM containing QSHX at final concentrations of 4, 2, 1, 0.5, 0.25, 0.125 and $0.625 \mathrm{mg} / \mathrm{ml}$ 
respectively in 6-well plates $\left(5 \times 10^{5}\right.$ cells/well), the supernatant were collected by centrifugation $\left(500 \mathrm{x} \mathrm{g}\right.$ for $6 \mathrm{~min}$ at $\left.4^{\circ} \mathrm{C}\right)$ and APN was measured using ELISA kits (ab108784; Abcam) according to the protocol provided by the manufacturer. Three parallel wells were set up for each concentration. In addition, the adipocytes were cultured with the optimal QSHX concentration, which resulted in the highest level of APN in above ELISA assay for $24 \mathrm{~h}$. The expression levels of HMW APN, Akt, P-Akt, FOXO1, P-FOXO1 and DsbA-L were then examined in the primary adipocytes using western blot analysis. This experiment was performed three times independently.

Statistical analysis. The data are expressed as the mean \pm standard deviation and were analyzed using SPSS version 17.0 (SPSS, Inc., Chicago, IL, USA). The comparisons were performed using one-way analysis of variance. $\mathrm{P}<0.05$ was considered to indicate a statistically significant difference.

\section{Results}

QSHX improves the general condition of rats. During the experiments performed in the present study, the rats in the normal control group were quiet and compliant, with normal hair and normal feeding. The rats in the model control group and treatment group were agitated and aggressive, and exhibited dark hair with hair loss, accompanied with a decrease in feeding from week 4 . The changes in the treatment group recovered gradually from week 13 . At the end of the experiment, eight rats had survived in each group, respectively.

QSHX decreases the body weight and liver indices of rats. No significant difference in body weight was observed between the rats prior to treatment in the treatment group and those in the model control group $(280.12 \pm 20.13$, vs. $273.33 \pm 19.35 \mathrm{~g}$, respectively; $\mathrm{P}=0.503$ ), however, the body weight in the treatment group prior to treatment was significantly increased, compared with that of rats in the normal control group $(280.12 \pm 20.13$. vs. $231.24 \pm 18.60$ g respectively; $\mathrm{P}<0.001)$. Following treatment, the body weight in the treatment group was significantly lower, compared with that in the model control group $(263.33 \pm 9.60$, vs. $336.33 \pm 35.72 \mathrm{~g}$, respectively; $\mathrm{P}<0.001$ ), as was the liver index (3.21 \pm 0.14 , vs. $3.56 \pm 0.24 \%$, respectively; $\mathrm{P}=0.006$ ). However, no significant difference was observed between body wright in the treatment group and that in the normal control group (263.33 \pm 9.60 , vs. $276.33 \pm 22.54$ g, respectively; $\mathrm{P}=0.156$ ), which was also true of liver index $(3.21 \pm 0.14$, vs. $3.14 \pm 0.24 \%$, respectively; $\mathrm{P}=0.488$ ) as shown in Fig. 1.

QSHX decreases serum ALT, AST and TG, and increases APN in NAFLD rats. Following treatment for 8 weeks, the levels of AST, ALT and TG in the model group were significantly increased $(27.33 \pm 4.55$, vs. $16.56 \pm 2.39 \mathrm{U} / 1 ; 53.57 \pm 21.18$ vs. $27.22 \pm 3.58 \mathrm{U} / 1$ and $1.40 \pm 0.50$, vs. $0.70 \pm 0.13 \mathrm{mmol} / 1$, respectively, compared with levels in the normal control group $(\mathrm{P}<0.001, \mathrm{P}<0.004$ and $\mathrm{P}<0.002$, respectively). The levels of AST, ALT and TG in the treatment group were significantly decreased, compared with those in the model control group (13.80 \pm 3.25 , vs. $27.33 \pm 4.55 \mathrm{U} / 1 ; 34.17 \pm 9.19$, vs. $53.57 \pm 21.18 \mathrm{U} / 1$ and $0.79 \pm 0.34$, vs. $1.40 \pm 0.50 \mathrm{mmol} / \mathrm{l}$, respectively; $\mathrm{P}<0.001$, $\mathrm{P}<0.032$ and $\mathrm{P}<0.013)$. The results of the ELISA indicated


Figure 1. QSHX decreases the body weight and liver index of rats. ${ }^{* *} \mathrm{P}<0.01$,


group; $\mathrm{M}$, model control group; $\mathrm{T}$, treatment group.
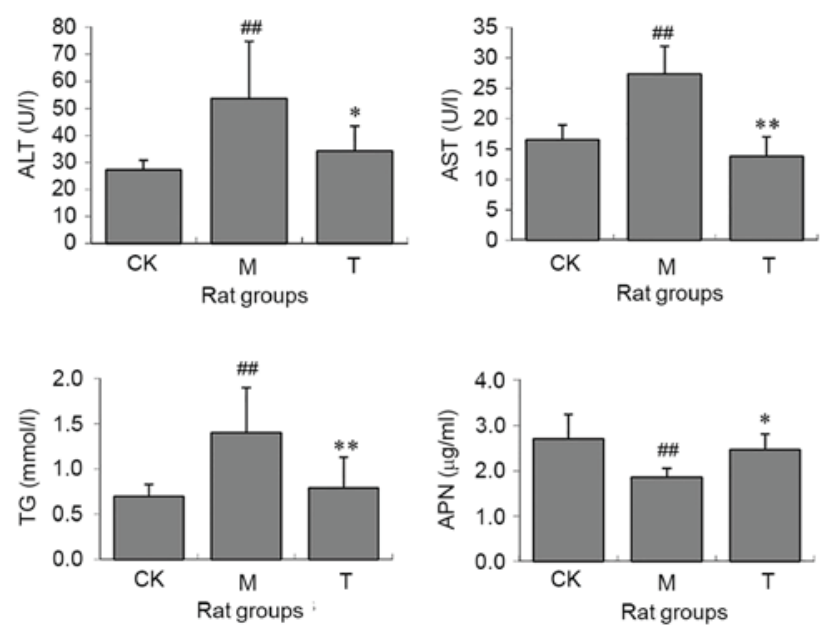

Figure 2. QSHX decreases serum ALT, AST, TG, and increases APN in rats with non-alcoholic fatty liver disease. ${ }^{*} \mathrm{P}<0.05$, $\mathrm{T}$ group vs. $\mathrm{M}$ group; ${ }^{* *} \mathrm{P}<0.01, \mathrm{~T}$ group vs. $\mathrm{M}$ group; ${ }^{\# \#} \mathrm{P}<0.01, \mathrm{M}$ group vs. CK group. $\mathrm{CK}$, normal control group; $\mathrm{M}$, model control group; T, treatment group. ALT, alanine aminotransferase; AST, aspartate aminotransferase; TG, triglyceride; APN, adiponectin.

that the level of APN in the treatment group was also significantly increased, compared with that in model control group (2.47 \pm 0.34 , vs. $1.86 \pm 0.20 \mu \mathrm{g} / \mathrm{ml} ; \mathrm{P}=0.001)$. The level of APN in the model group was significantly lower, compared with that in the normal control group $(1.86 \pm 0.2$, vs. $2.71 \pm 0.54 \mu \mathrm{g} / \mathrm{ml}$; $\mathrm{P}<0.001$; Fig. 2).

QSHX reduces hepatic steatosis in NAFLD rats. Under the microscope, the HE staining in the normal control group (Fig. 3A) showed normal liver lobules with a clear structure, no fat vacuoles and dark colored plasma. In the model control group (Fig. 3B), the liver lobules were not clear in structure, the liver was filled with fat vacuoles of different sizes (fat drops), the collagen around blood vessels was increased and there were few inflammatory cells around the bile conduct. In the treatment group, the liver showed lobules with a clear structure, fat drops of different sizes, increased collagen around blood vessels and few inflammatory cells around the bile conduct (Fig. 3C). Following 8 weeks of treatment, the hepatic steatosis in the treatment group significantly improved and the steatosis score was significantly decreased, compared with the model group $(1.79 \pm 0.44$, vs. $2.57 \pm 0.87$; $\mathrm{P}=0.04$; Fig. 4). 
A

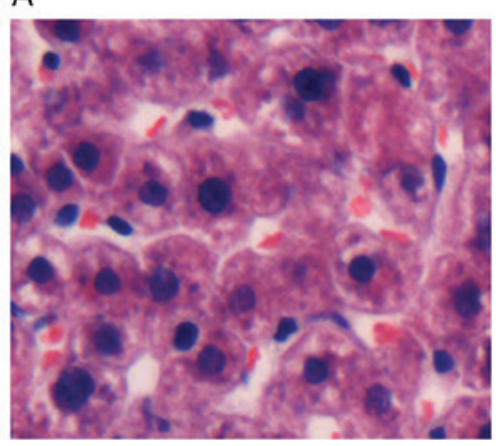

B

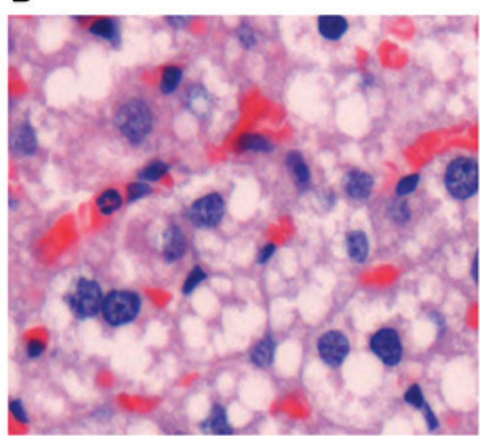

C



Figure 3. Hematoxylin and eosin staining of liver structures in different groups of rats. Images of staining under a microscope (magnification, x200). (A) normal control group; (B) model control group; (C) treatment group.

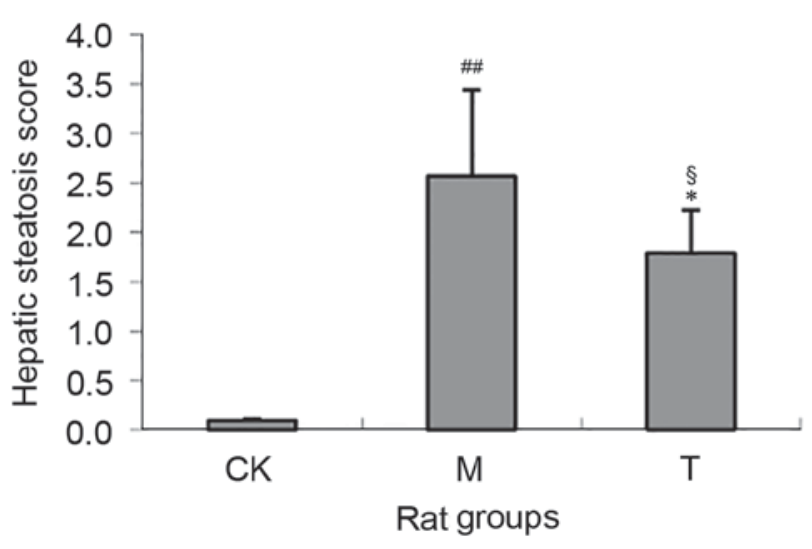

Figure 4. Hepatic steatosis scores in rats with non-alcoholic fatty liver disease. ${ }^{*} \mathrm{P}<0.05, \mathrm{~T}$ group vs. $\mathrm{M}$ group; ${ }^{\circledR} \mathrm{P}<0.05, \mathrm{~T}$ group vs, CK group; ${ }^{\# \#} \mathrm{P}<0.01, \mathrm{M}$ group vs. CK group. $\mathrm{CK}$, normal control group; $\mathrm{M}$, model control group; T, treatment group.

Effects of QSHX on the Akt/FOXO1 pathway, DsbA-L and $H M W$ APN in adipose tissue. The expression of Akt, p-Akt, FOXO1, p-FOXO1, DsbA-L and HMW APN in adipose tissue are shown in Fig. 5A. The ratio of target protein to GAPDH optical density (OD) was used to analyze the relative expression abundance. The results of the western blot analysis indicated that the expression levels of p-Akt and p-FOXO1 in the treatment group were inhibited by QSHX, the OD value ratios were lower, compared with those in the model control group $(0.11 \pm 0.03$, vs. $0.38 \pm 0.08$ and $0.24 \pm 0.05$, vs. $0.54 \pm 0.09$, respectively; $\mathrm{P}<0.001)$. However, the expression levels of p-Akt and p-FOXO1 were significantly higher in the model control group, compared with those in the normal control group $(0.38 \pm 0.08$, vs. $0.28 \pm 0.07$ and $0.54 \pm 0.09$, vs. $0.44 \pm 0.10$, respectively; $\mathrm{P}<0.005$ and $\mathrm{P}<0.001)$. The expression levels of DsbA-L and HMW APN in the treatment group were increased by QSHX, with higher OD value ratios, compared with those in the model control group $(0.58 \pm 0.14$, vs. $0.14 \pm 0.04$ and $0.14 \pm 0.02$, vs. $0.10 \pm 0.03$, respectively; $\mathrm{P}<0.001$ and $\mathrm{P}<0.036$ ). The expression levels of DsbA-L and HMW APN in the model control group were lower, compared with those in the normal control group $(0.14 \pm 0.04$ vs. $0.53 \pm 0.10,0.10 \pm 0.03$ vs. $0.17 \pm 0.05$ respectively; $\mathrm{P}<0.001$ and $\mathrm{P}<0.001$ ). No significant difference was found in the expression of Akt between the normal control group, model control group and treatment group (data not shown), nof was any difference found in the expression of FOXO1 between the normal group and model group (1.20 \pm 0.16 , vs. $1.22 \pm 0.11 ; \mathrm{P}=0.803)$. However, QSHX significantly inhibited its expression, with OD value ratios of $0.48 \pm 0.12$ and $1.22 \pm 0.11$ in the treatment group and model control group, respectively ( $\mathrm{P}<0.001$; Fig. 5B).

Effect of QSHX on the secretion of APN in primary adipocyte supernatant. The primary adipocytes were incubated with various concentrations of QSHX for $24 \mathrm{~h}$. The results of APN levels are showed in Fig. 6, The lower concentrations of QSHX $(0.0625-0.50 \mathrm{mg} / \mathrm{ml})$ increased the secretion of APN, which peaked at $0.5 \mathrm{mg} / \mathrm{ml}$. However, from a concentration $1 \mathrm{mg} / \mathrm{ml}$, the secretion of APN was progressively decreased with increasing concentration of QSHX, and the highest concentration $(4 \mathrm{mg} / \mathrm{ml})$ inhibited the secretion of APN. Therefore, the concentration of $0.5 \mathrm{mg} / \mathrm{ml}$ was selected for the subsequent experiment.

Effect of QSHX on the expression of HMW APN, Akt, p-Akt, FOXO1, p-FOXO1 and DsbA-L in primary adipocytes. Primary adipocytes were collected following incubation with $0.5 \mathrm{mg} / \mathrm{ml}$ QSHX for $24 \mathrm{~h}$. A blank control was set up. The expression levels of HMW APN, Akt, p-Akt, FOXO1, p-FOXO1 and DsbA-L were measured using western blot analysis. As shown in Fig. 7, the levels of HMW APN and DsbA-L were increased, whereas the levels of FOXO1 and p-FOXO1 were decreased following treatment with $0.5 \mathrm{mg} / \mathrm{ml}$ QSHX.

\section{Discussion}

It is advised in clinical practice that patients with NASH or who are at risk from NASH be treated with exercise therapy and diet supplementation with vitamin $\mathrm{E}$ or pentoxifylline (6). However, drugs targeting insulin-resistance, including thiazolidinedione and melbine, antioxidants, including vitamin $\mathrm{E}$, anti-fibrotic drugs and pentoxifylline, are all considered experimental treatments as they are not supported by double-blind control experiments. Currently, effective anti-NAFLD/NASH drugs are in the development stage.

APN is one of the adipokines secreted by adipocytes (7). Insulin-resistant experiments in animals and clinical studies have indicated that recombinant APN or whole length of 
A

GAPDH

$\begin{array}{lll}\mathrm{CK} & \mathrm{M} & \mathrm{T}\end{array}$

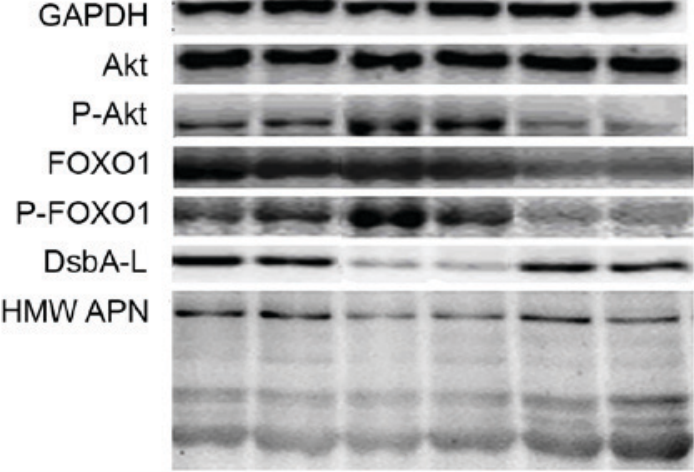

B
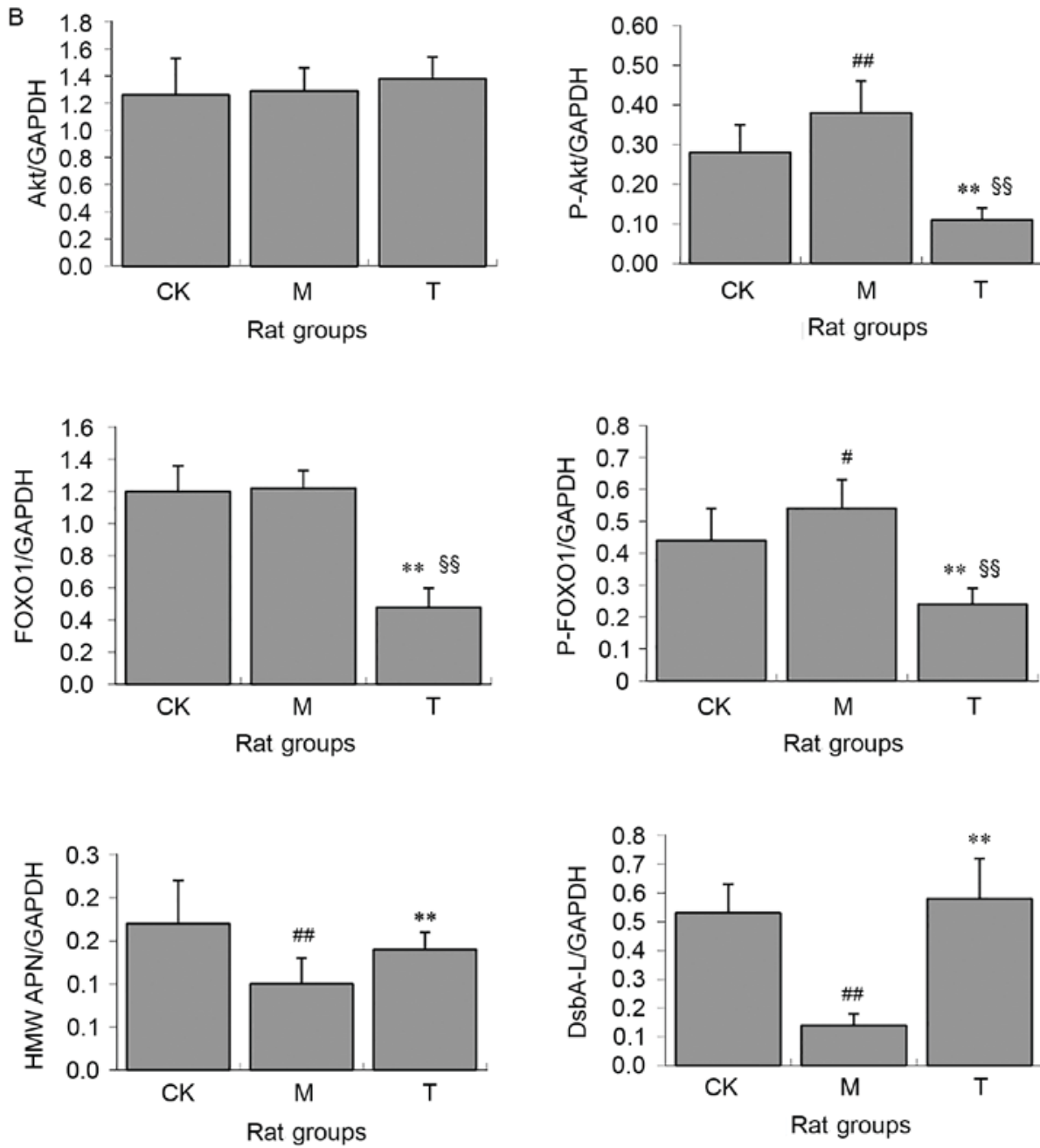

Figure 5. Expression of proteins in adipose tissue analyzed using western blot analysis. Results of the western blot analysis are showed in graph. (A and B) Optical density value ratios of target protein to GAPDH are showed in bar graphs. ${ }^{* *} \mathrm{P}<0.01, \mathrm{~T}$ group vs. $\mathrm{M}$ group; ${ }^{\$ \S} \mathrm{P}<0.01, \mathrm{~T}$ group vs. CK group; ${ }^{\sharp} \mathrm{P}<0.05, \mathrm{M}$ group vs. CK group; ${ }^{\# \#} \mathrm{P}<0.01, \mathrm{M}$ group vs. CK group. CK, normal control group; $\mathrm{M}$, model control group; T, treatment group; HMW APN, high molecular weight adiponectin; FOXO1, forkhead box O protein 1; DsbA-L, disulfide-bond A oxidoreductase-like protein; p-, phosphorylated.

endogenous APN can decrease the level of blood sugar and accelerate the removal of plasma lipids following consumption of a high-fat diet $(8,9)$. The levels of APN in serum and liver tissues, and the level of APN receptor in the liver tissues of patients with NAFLD are decreased; the decrease in serum APN is more marked in patients with NASH, compared with patients with single fatty liver disease, and are negatively correlated with the level of ALT $(10,11)$. Therefore, APN may be a predicator of the severity of liver disease in patients with
NAFLD/NASH $(10,11)$. APN can inhibit NAFLD through increasing the oxidation of fat acid, reducing fat content (12), inhibiting the expression of profibrotic factors, and the activation, proliferation and migration of hepatic stellate cells (HSCs) (13), and promoting the apoptosis of activated HSCs (14). APN has a similar structure to tumor necrosis factor- $\alpha$ (TNF- $\alpha$ ) and shares the same signaling pathway, therefore, APN can antagonize TNF- $\alpha$ to reduce liver inflammation (15). In the present study, a high-fat and high-sugar diet was administered 


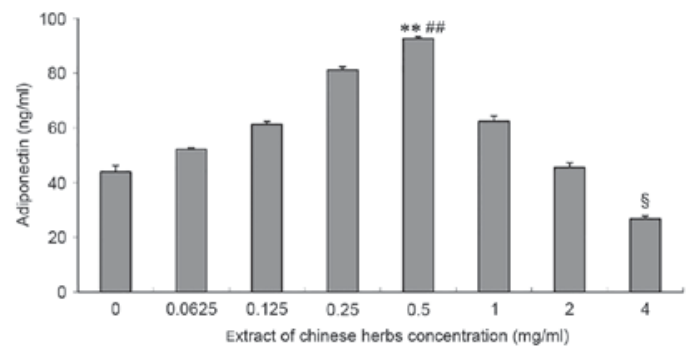

Figure 6. Effect of QSHX on secretion of APN in the supernatant of primary adipocytes. Lower concentrations of QSHX $(0.0625-0.50 \mathrm{mg} / \mathrm{ml})$ increased the secretion of APN and reached a peak at $0.5 \mathrm{mg} / \mathrm{ml}$. At concentrations of $1 \mathrm{mg} / \mathrm{ml}$ and above, the secretion of APN decreased gradually with increased concentration of QSHX, with the highest concentration of $4 \mathrm{mg} / \mathrm{ml}$ inhibiting the secretion of APN. ${ }^{* *} \mathrm{P}<0.01,0.5 \mathrm{mg} / \mathrm{ml}$ vs. $0 \mathrm{mg} / \mathrm{ml} ;{ }^{\# \#} \mathrm{P}<0.01,0.5 \mathrm{mg} / \mathrm{ml}$ vs. $0.25 \mathrm{mg} / \mathrm{ml} ;{ }^{\circledR} \mathrm{P}<0.05,4 \mathrm{mg} / \mathrm{ml}$ vs. $0 \mathrm{mg} / \mathrm{ml}$. APN, adiponectin.

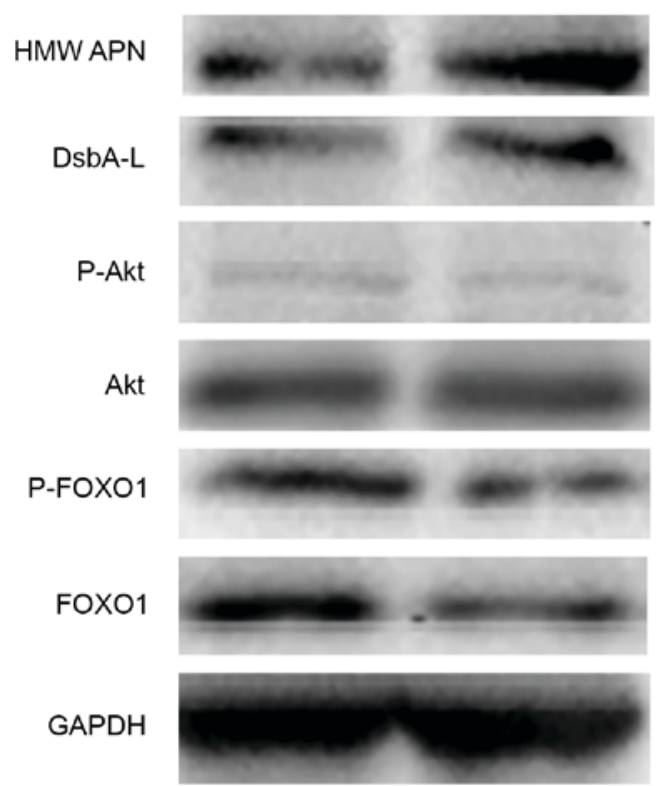

Figure 7. Effect of QSHX on expression levels of HMW APN, Akt, p-Akt, FOXO1, p-FOXO1 and DsbA-L in primary adipocytes. Expression levels of HMW APN and DsbA-L were increased, and those of FOXO1 and p-FOXO1 were decreased by $0.5 \mathrm{mg} / \mathrm{ml}$ QSHX. CK, blank control, T, QSHX group; HMW APN, high molecular weight adiponectin; FOXO1, forkhead box O protein 1; DsbA-L, disulfide-bond A oxidoreductase-like protein; p-, phosphorylated.

to establish an NAFLD model in rats, and these NAFLD rats were treated with QSHX. The results indicated that the plasma levels of APN in the NAFLD rats were significantly lower, compared with those in normal animals, whereas treatment with QSHX increased the levels of APN and reduced hepatic steatosis. Therefore, it was hypothesized that the improvement of hepatic steatosis by QSHX occurred through promoting the expression of APN.

In the circulation, at least three complexes of APN exist, including trimer, six dimer and 12-18 monomer molecular weight forms (HMW) (16). HMW APN shows significant correlation with insulin sensitivity and its proportion is a key factor in insulin tolerance (17). DsbA-L is expressed in the liver, kidneys, pancreas and heart, however, its expression is highest in fat tissue (18). Liu et al (19) confirmed that DsbA-L is a novel molecular chaperone and component of the complete
APN complex for the synthesis and secretion of APN, and is involved in the formation of HMW APN. It has been found that DsbA-L inhibits the downregulation of APN by endoplasmic reticulum (ER) stress in 3T3-L1 cells, primarily at the protein level (20). Therefore, the present study was performed at the protein level. Wang et al (4) found that the Akt signal is important in modulating the expression of DsbA-L. The treatment of 3T3-L1 cells with the specific Akt inhibitor, AKTI, inhibits the phosphorylation of Akt and downstream FOXO1, but increases the expression of DsbA-L and APN (21). As a direct substrate of Akt and nuclear transcriptional factor, FOXO1 negatively modulates downstream target genes. Activated Akt (p-Akt) can phosphorylate FOXO1, resulting in the translocation of FOXO1 to the cytoplasm from the nucleus and loss of capability to activate transcription (22). FOXO1 has been confirmed to be involved in the negative modulation of APN (22). The results of the present study indicated that the expression levels of p-Akt and p-FOXO1 were increased in the NAFLD rats, which may result in a decrease of DsbA-L and HMW APN. In the animals treated with QSHX, the expression levels of FOXO1, p-FOXO1 and p-Akt were decreased, whereas those of DsbA-L and HMW APN were increased. In the primary adipocytes of the rats, QSHX also promoted the expression levels of DsbA-L and HMW APN, and inhibited the expression and activation of FOXO1. These results indicated that QSHX inhibits the FOXO1 pathway to increase the expression ofHMW APN, which may be one of the mechanisms underlying the effect of QSHX in improving NAFLD.

In conclusion, the present study indicated that QSHX significantly inhibited hepatic steatosis in the NAFLD rats through inhibiting activation of the FOXO1 signal, increasing the expression of DsbA-L and HMW APN. These results provide novel theoretic evidence for QSHX in treating metabolic diseases, including NAFLD, providing a novel therapeutic option for clinicians to treat NAFLD.

\section{Acknowledgements}

This study was supported by the National Natural Science Foundation of China (grant no. 81360596), the Guangxi Natural Science Foundation (grant no. 2014GXNSFDA118029) and the Guangxi BaGui Scholars Program Foundation.

\section{References}

1. Karim MF, Al-Mahtab M, Rahman S and Debnath CR: Non-alcoholic fatty liver disease (NAFLD)-a review. Mymensingh Med J 24: 873-880, 2015.

2. Review T, LaBrecque DR, Abbas Z, Anania F, Ferenci P, Khan AG, Goh KL, Hamid SS, Isakov V, Lizarzabal M, et al: World Gastroenterology Organisation global guidelines: Nonalcoholic fatty liver disease and nonalcoholic steatohepatitis. J Clin Gastroenterol 48: 467-473, 2014.

3. Jin D, Sun J, Huang J, Yu X, Yu A, He Y, Li Q and Yang Z: Peroxisome proliferator-activated receptor $\gamma$ enhances adiponectin secretion via up-regulating DsbA-L expression. Mol Cell Endocrinol 411: 97-104, 2015.

4. Wang A, Liu M, Liu X, Dong LQ, Glickman RD, Slaga TJ, Zhou $\mathrm{Z}$ and Liu F: Up-regulation of adiponectin by resveratrol: The essential roles of the Akt/FOXO1 and AMP-activated protein kinase signaling pathways and DsbA-L. J Biol Chem 286: 60-66, 2011.

5. Liu X, Tu Y and Zhang H: 40 cases of nonalcoholic fatty liver disease were treated by QushiHuoxue Chinese herbs. Shaanxi J Traditional Chin Med 34: 16-18, 2013 (In China). 
6. Watanabe S, Hashimoto E, Ikejima K, Uto H, Ono M, Sumida Y, Seike M, Takei Y, Takehara T, Tokushige K, et al: Evidence-based clinical practice guidelines for nonalcoholic fatty liver disease/nonalcoholic steatohepatitis. J Gastroenterol 50: 364-377, 2015.

7. Yoda-Murakami M, Taniguchi M, Takahashi K, Kawamata S, Saito K, Choi-Miura NH and Tomita M: Change in expression of GBP28/adiponectin in carbon tetrachloride-administrated mouse liver. Biochem Biophys Res Commun 285: 372-377, 2001.

8. Xu A, Wang Y, Keshaw H, Xu LY, Lam KS and Cooper GJ: The fat-derived hormone adiponectin alleviates alcoholic and nonalcoholic fatty liver diseases in mice. J Clin Invest 112: 91-100, 2003.

9. Kubota N, Terauchi Y, Yamauchi T, Kubota T, Moroi M, Matsui J, Eto K, Yamashita T, Kamon J, Satoh H, et al: Disruption of adiponectin causes insulin resistance and neointimal formation. J Biol Chem 277: 25863-25866, 2002.

10. Vuppalanchi R, Marri S, Kolwankar D, Considine RV and Chalasani N: Is adiponectin involved in the pathogenesis of nonalcoholic steatohepatitis? A preliminary human study. J Clin Gastroenterol 39: 237-242, 2005.

11. Musso G, Gambino R, Biroli G, Carello M, Fagà E, Pacini G, De Michieli F, Cassader M, Durazzo M, Rizzetto M and Pagano G: Hypoadiponectinemia predicts the severity of hepatic fibrosis and pancreatic Beta-cell dysfunction in nondiabetic nonobese patients with nonalcoholic steatohepatitis. Am J Gastroenterol 100 2438-2446, 2005.

12. You M, Matsumoto M, Pacold CM, Cho WK and Crabb DW: The role of AMP-activated protein kinase in the action of ethanol in the liver. Gastroenterology 127: 1798-1808, 2004

13. Arita Y, Kihara S, Ouchi N, Maeda K, Kuriyama H, Okamoto Y, Kumada M, Hotta K, Nishida M, Takahashi M, et al: Adipocyte-derived plasma protein adiponectin acts as a platelet-derived growth factor-BB-binding protein and regulates growth factor-induced common postreceptor signal in vascular smooth muscle cell. Circulation 105: 2893-2898, 2002.
14. Ding X, Saxena NK, Lin S, Xu A, Srinivasan S and Anania FA: The roles of leptin and adiponectin: A novel paradigm in adipocytokine regulation of liver fibrosis and stellate cell biology. Am J Pathol 166: 1655-1669, 2005.

15. Kadowaki $\mathrm{T}$ and Yamauchi T: Adiponectin and adiponectin receptors. Endocr Rev 26: 439-451, 2005.

16. Goto M, Goto A, Morita A, Deura K, Sasaki S, Aiba N, Shimbo T, Terauchi Y, Miyachi M, Noda M, et al: Low-molecular-weight adiponectin and high-molecular-weight adiponectin levels in relation to diabetes. Obesity 22: 401-407, 2014.

17. Simpson F and Whitehead JP: Adiponectin-it's all about the modifications. Int J Biochem Cell Biol 42: 785-788, 2010.

18. Ladner JE, Parsons JF, Rife CL, Gilliland GL and Armstrong RN: Parallel evolutionary pathways for glutathione transferases: Structure and mechanism of the mitochondrial class kappa enzyme rGSTK1-1. Biochemistry 43: 352-361, 2004.

19. Liu M, Zhou L, Xu A, Lam KS, Wetzel MD, Xiang R, Zhang J, Xin X, Dong LQ and Liu F: A disulfide-bond A oxidoreductase-like protein (DsbA-L) regulates adiponectin multimerization. Proc Natl Acad Sci USA 105: 18302-18307, 2008.

20. Zhou L, Liu M, Zhang J, Chen H, Dong LQ and Liu F: DsbA-L alleviates endoplasmic reticulum stress-induced adiponectin downregulation. Diabetes 59: 2809-2816, 2010.

21. Gabrielsen JS, Gao Y, Simcox JA, Huang J, Thorup D, Jones D, Cooksey RC, Gabrielsen D, Adams TD, Hunt SC, et al: Adipocyte iron regulates adiponectin and insulin sensitivity. J Clin Invest 122: 3529-3540, 2012.

22. Nakae J, Cao Y, Oki M, Orba Y, Sawa H, Kiyonari H, Iskandar K, Suga K, Lombes M and Hayashi Y: Forkhead transcription factor FoxO1 in adipose tissue regulates energy storage and expenditure. Diabetes 57: 563-576, 2008. 